\title{
Développement de détecteurs en diamant CVD pour la caractérisation de sources impulsionnelles UV-X
}

\author{
B. Guizard, P. Bergonzo, C. Mer, A. Brambilla, D. Tromson et F. Foulon
}

LETI, CEA-Technologies Avancées, DEIN/SPE, CEA/Saclay, 91191 Gif-sur-Yvette, France

\begin{abstract}
Résumé : De par ses propriétés électriques et physico-chimiques, le diamant est un matériau remarquable pour la fabrication de détecteurs de rayonnements. Des détecteurs ont récemment été développés au laboratoire pour des applications de métrologie des rayonnements. Dans le cadre spécifique de la caractérisation de sources impulsionnelles, des dispositifs permettant le contrôle de l'intensité et de la dose sous impulsions sub-nanosecondes de rayonnements UV et $X$ ont été fabriqués. Après présentation des principes expérimentaux, cette étude décrit le mode de fonctionnement et leur utilisation en particulier pour des mesures de dose sur l'installation de radiographie des systèmes dynamiques hyper rapides (AIRIX) et pour la métrologie de trains d'impulsions.
\end{abstract}

\section{INTRODUCTION}

Depuis 1993, le Département d'Électronique et d'Instrumentation Nucléaire (DEIN) du LETI-CEA/Saclay développe des détecteurs de radiations à base de diamant polycristallin synthétisé par dépôt chimique en phase vapeur assisté par plasma micro-onde (MWCVD) [1].

Cet article met en évidence les capacités du diamant pour la détection de faisceaux $\mathrm{X}$ de très haute intensité et pour la métrologie de trains d'impulsions rapides (subnanoseconde). Ces détecteurs présentent un grand intérêt pour la caractérisation de nouvelles sources de photons, telles que les lasers VUV, femtoseconde, et le rayonnement synchrotron de troisième génération.

\section{FABRICATION ET PRINCIPE DE DÉTECTION}

Les propriétés. intrinsèques remarquables du diamant permettent son utilisation pour la fabrication de détecteurs de rayonnements. C'est un semi-conducteur à grande largeur de bande interdite $(5,5 \mathrm{eV})$, qui peut fonctionner à haute température et qui présente une forte résistance aux rayonnements. En outre, il permet d'obtenir une très bonne discrimination entre le rayonnement UV lointain $(\lambda<225 \mathrm{~nm})$ et les photons du domaine visible (effet solar blind). De plus, la faible durée de vie des porteurs permet la réalisation de détecteurs ultra-rapides $(<50 \mathrm{ps})$.

Les détecteurs sont élaborés au DEIN par la technique de dépôt CVD assisté par plasma micro-onde à $2,45 \mathrm{GHz}$. Les gaz employés sont le méthane et l'hydrogène à des pressions variant de 50 à 150 mbar. Lors du dépôt, la dissociation du méthane entraîne la création d'espèces actives carbonées. La réaction de ces espèces avec la surface chauffée $\left(800^{\circ} \mathrm{C}\right)$ conduit à la formation d'un film mince de diamant dont la structure est polycristalline. Les substrats de diamant réalisés ont des géométries variables allant de disques de 5 centimètres de diamètre à des carrés de $5 \times 5 \mathrm{~mm}^{2}$. L'épaisseur est comprise entre un et plusieurs centaines de microns, suivant l'application visée.

Le dispositif de détection est constitué d'un échantillon de diamant non dopé sur lequel sont déposés deux contacts électriques qui permettent de polariser le détecteur. Lorsqu'une impulsion radiative traverse le détecteur, elle génère des paires électron-trous qui, en dérivant sous l'effet du champ appliqué produisent une impulsion de courant aux bornes du détecteur. À partir de l'ajustage des conditions de synthèse du diamant, il a été possible d'optimiser les caractéristiques de réponse de ces détecteurs, afin de permettre la mesure d'impulsions ultra-rapides, en vue de leur application pour la métrologie de la dose et de la distribution temporelle des sources de rayonnements. 


\section{MESURE DE DOSE SUR SYSTÈME HYPER RAPIDES}

Des détecteurs opérationnels ont été élaborés au DEIN pour l'étalonnage de l'installation AIRIX (Accélérateur à Induction Radiographique pour Imagerie X). Ce dernier est un accélérateur linéaire d'électrons de fortes énergies et de très haute intensité. L'impulsion, d'une durée d'environ 100 ns, délivre un rayonnement permettant de réaliser des clichés radiographiques d'objets à dynamique ultra-rapide (explosion de charges de matériaux).

Le DEIN a développé un dispositif comprenant cinq détecteurs ; quatre d'entre eux sont disposés en carré et permettent de contrôler le positionnement du faisceau et de mesurer sa distribution spatiale. Le dernier, placé au centre, mesure l'intensité et la forme temporelle du faisceau.

En fonction de la dose reçue sur le détecteur (de 100 à $10000 \mathrm{Rad}$ ), la réponse de ce dernier présente une caractéristique parfaitement linéaire (figure 1). Ce dispositif permet donc l'étalonnage en intensité du faisceau d'électrons. La résolution temporelle du détecteur est inférieure à $1 \mathrm{~ns}$ et sa tenue aux rayonnements supérieure à $70 \mathrm{Mrad}$. Il répond parfaitement aux exigences de l'installation.

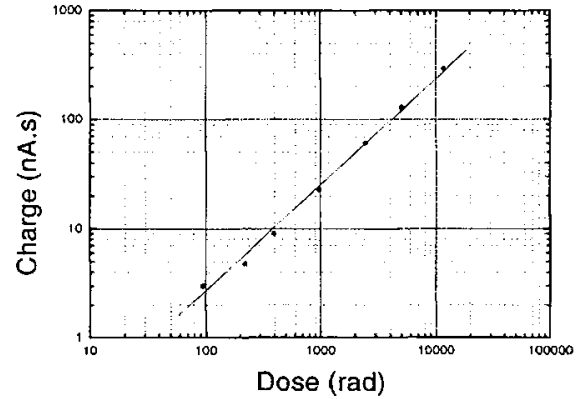

Figure 1 : Réponse du détecteur en fonction de la dose envoyée

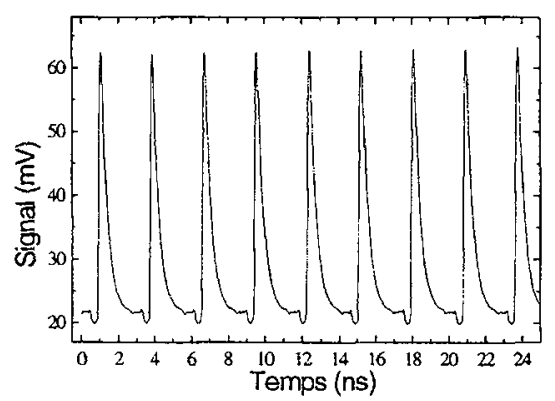

Figure 2 : Forme du train d'impulsion X à l'ESRF

\section{MÉTROLOGIE D'IMPULSIONS ULTRA-RAPIDES}

Il s'agit ici de pouvoir mesurer la distribution en intensité de trains d'impulsions à très forts taux de répétition en particulier ceux issus du rayonnement synchrotron de I'ESRF : la forme du train d'impulsions $X$ est ici constituée de 660 impulsions de 40 ps espacées de $2,8 \mathrm{~ns}$. Leur énergie s'étale de 5 à $20 \mathrm{KeV}$. À partir de l'optimisation du procédé de synthèse du diamant, il a été possible de réduire le temps de récupération du détecteur à des valeurs inférieures à $70 \%(90 \%-10 \%)$.

La figure 2 représente la forme d'un train d'impulsions enregistrée avec un tel détecteur. On constate que ce dernier permet la discrimination parfaite des impulsions. Les variations d'intensités relevées sont alors directement superposables aux caractéristiques machines attendues. Ces détecteurs présentent donc l'avantage d'être petits, peu onéreux et simples d'utilisation (par rapport à celle d'une caméra à balayage de fente).

\section{CONCLUSION}

Des photodétecteurs en diamant CVD ont été spécifiquement développés pour la caractérisation de faisceaux UV-X $(10 \mathrm{eV}$ à $10 \mathrm{KeV})$. Ils répondent parfaitement aux exigences de rapidité de réponse et de résistance aux rayonnements des nouvelles sources. Ils présentent un réel avantage pour la métrologie d'impulsions ultra-rapides ou à fort taux de répétition, telles que celles émises par les rayonnements synchrotrons.

\section{Référence}

1. P. Bergonzo, A. Brambilla, D. Tromson, C. Mer, B. Guizard, F. Foulon, Appl Surf Sci 154 (2000) 179 